\title{
DEVICE LEVEL ELECTROTHERMAL ANALYSIS OF INTEGRATED RESISTORS
}

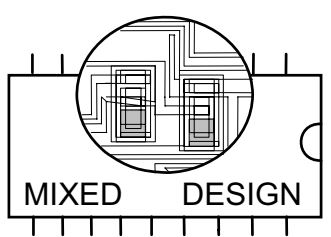

MIXDES 2007

Ciechocinek, POLAND

\section{1 - 23 June 2007}

B. VermeERSCh, G. De Mey

GHENT UNIVERSITY, BELGIUM

KEYWORDS: Electrothermal analysis, Resistor, Thermal runaway

ABSTRACT: This paper presents the electrothermal simulation of integrated thin film resistors. Both the thermal and electrical problem is tackled by a semi-analytical method, without the need of generating an equivalent distributed network. As the electrical conductivity is temperature dependent, self-heating of the resistor will alterate the current distribution, leading to a non-uniform power dissipation. This then provokes a change of the temperature distribution, explaining the electrothermal coupling. Examples are given for various practical resistor designs. After a few iterations stable values for the electrical and thermal resistance and temperature and power distributions are obtained. The results show that even if one would anticipate the self-heating process based on an estimated average temperature, the behaviour will still deviate from the original design. This is caused entirely by the non-uniformity of the distributions inside the component.

\section{INTRODUCTION}

It is well known that many electrical material properties depend on the temperature, especially in semiconductors. Therefore the electrical characteristics of common devices such as diodes and transistors are temperature dependent as well. Heating of a component, either self-induced or caused by surrounding devices and environmental conditions, will change its electric behaviour. The alteration of the voltage and/or current gives rise to a modified power dissipation, which finally influences the temperature. Hence the electrical and thermal behaviour of the devices are coupled in a tight and rather complex way.

In integrated circuits (ICs), these couplings are very likely to occur since a large number of heat-dissipating elements are placed on a small area. Other authors earlier published a PSPICE/TULSOFT based simulator for electrothermal analysis of various IC designs [1]. In contrast however to this and other works in the literature considering an entire circuit, we will focus on only one single component, namely a resistor. In such a case the electrothermal coupling arises from the temperature dependence of the electrical conductivity $\sigma$. This leads to a non-uniform current and power dissipation distribution. The considered configuration is shown in Fig. 1.

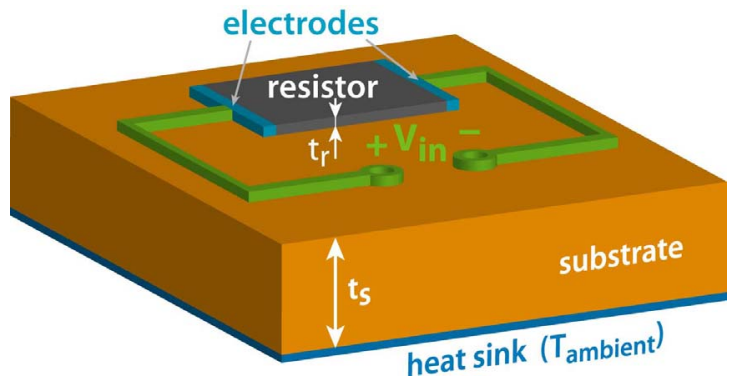

Figure 1. Resistor integrated on rear-cooled substrate
A resistor is made of a thin film with thickness $t_{r}$. A voltage $V_{i n}$ is applied between electrodes making contact in two edges of the material film. The resistor is integrated on top of a substrate with thickness $t_{s}$. The bottom of the substrate is kept at a constant temperature $T_{\text {ambient }}$, while we assume the upper surface as adiabatic (thermally isolated). Without affecting the generality, $T_{\text {ambient }}$ can be chosen as zero, thus all following temperatures denote values relative to ambient.

In the following we will present a method for solving the electrical and thermal problem for the layout sketched in Fig. 1. Electrothermal analysis can then be performed by alternate application of the two solvers. Convergence is obtained after no more than five iterations, as illustrated by two practical resistor designs. Finally a thermal runaway phenomenon is demonstrated.

\section{SOLUTION PROCEDURES}

\section{Electrical Solver}

First of all, variations along the thickness of the material film ( $z$-direction) will be neglected for both electrical and thermal distributions. This is reasonable as long as the lateral dimensions ( $x$ - and $y$-directions) are relatively large compared to $t_{r}$. Due to this assumption all relevant quantities are two-dimensional, e.g. for the voltage we have $\phi(x, y)$. The electric field (in $\mathrm{V} / \mathrm{m}$ ) is then given by:

$$
\vec{E}(x, y)=-\vec{\nabla} \phi(x, y)
$$

The resistor material is characterized by an electrical conductivity $\sigma(x, y)$ (in $\Omega^{-1} \mathrm{~m}^{-1}$ ). Note that $\sigma$ should be seen as $x$ and $y$ dependent, since it is influenced by the (non-uniform) temperature field (see the section on the thermal solver). The conductivity relates the electric field to the current density $\vec{J}$ (in $\mathrm{A} / \mathrm{m}^{2}$ ):

$$
\vec{J}(x, y)=\sigma(x, y) \vec{E}(x, y)
$$


As we are limiting ourselves to steady state situations, the continuity equation simplifies to:

$$
\vec{\nabla} \cdot \vec{J}(x, y)=0
$$

Using (1) and (2) we finally have:

$$
\vec{\nabla} \cdot[\sigma(x, y) \vec{\nabla} \phi(x, y)]=0
$$

In case of a uniform conductivity the Laplace equation $\nabla^{2} \phi=0$ is obtained, as expected.

Eq. (4) is a partial differential equation (PDE) for the voltage distribution inside the resistor. The boundary conditions are as follows:

- $\phi(x, y)=V_{\text {in }}$ at the positive electrode,

- $\phi(x, y)=0$ at the negative electrode, and

- $\frac{\partial \phi}{\partial n}=0$ along all other edges, where $\vec{n}$ is an outward pointing unit vector perpendicular to the segment.

A solution for $\phi$ can be obtained relatively easily with a numerical technique, e.g. using Matlab's PDEtool functions. The algorithms are based on a finite element method. The resistor layout is split into a mesh of triangles. After specifying the boundary conditions, and $\sigma$ for each triangle, the voltage in each node is calculated. We can now determine the volumetric power density $P_{V}$ (in $\left.\mathrm{W} / \mathrm{m}^{3}\right)$ :

$$
P_{V}(x, y)=\vec{J}(x, y) \cdot \vec{E}(x, y)
$$

and hence, combining (1) and (2):

$$
\begin{aligned}
P_{V}(x, y) & =\sigma(x, y)|\vec{\nabla} \phi(x, y)|^{2} \\
& =\sigma(x, y)\left[\left(\frac{\partial \phi}{\partial x}\right)^{2}+\left(\frac{\partial \phi}{\partial y}\right)^{2}\right]
\end{aligned}
$$

The equivalent surface power density $P$ (in $\mathrm{W} / \mathrm{m}^{2}$ ) dissipated in the thin film is then immediately found by simply multiplying $P_{V}$ with the resistor thickness $t_{r}$. This information is then passed to the thermal solver in order to calculate the temperature distribution, as explained below.

\section{Thermal Solver}

The substrate material is characterized by a thermal conductivity $k$ (in $\mathrm{W} / \mathrm{mK}$ ). To keep things manageable, we will at first neglect the temperature dependence of $k$. A full analysis requires an equation of the form (4) to be solved in the three-dimensional substrate volume. This approach would be very complicated and time consuming and will therefore not be used here. The temperature dependence may however be taken into account with an approximative perturbation of the temperature solution, as explained further.

Under the circumstances of a constant thermal conductivity the temperature distribution in the substrate satisfies:

$$
\nabla^{2} T(x, y, z)=0
$$

On the substrate top surface the resistor is dissipating power, with a density $P(x, y)$ as just obtained from the electrical simulation. Due to the assumption of $k$ being constant, the resulting temperature distribution can be calculated semi-analytically. The method is based on the Green's function for the semi-infinite 3-D space:

$$
G(\Delta x, \Delta y, \Delta z)=\frac{1}{2 \pi k r}
$$

with $r=\sqrt{(\Delta x)^{2}+(\Delta y)^{2}+(\Delta z)^{2}}$ the distance between the field point $(x, y, z)$ and source point $\left(x^{\prime}, y^{\prime}, z^{\prime}\right)$. As the Green's function is the fundamental solution of the heat equation, i.e. for a point source with unit power $\delta(x, y)$, the temperature for a distributed source can be found by superposition. In the denominator of $G$ a factor 2 instead of 4 occurs, due to the adiabatic boundary condition on top of the substrate. Note that (8) applies for a semi-infinite space, in other words it corresponds with $t_{s} \rightarrow \infty$. A bottom-cooled substrate with a finite thickness can be analyzed using the multiple reflection technique. As we only need the temperature inside the heat source (hence $\Delta z=0$ ), the following extended Green's function can be used:

$$
G^{*}(\Delta x, \Delta y)=\frac{1}{2 \pi k r_{0}}+2 \sum_{n=1}^{\infty} \frac{(-1)^{n}}{2 \pi k r_{n}}
$$

in which

$$
r_{n}=\sqrt{(\Delta x)^{2}+(\Delta y)^{2}+\left(2 n t_{s}\right)^{2}}
$$

Application of the superposition principle finally leads us to:

$$
T_{\text {source }}(x, y)=\iint_{\text {resistor }} P\left(x^{\prime}, y^{\prime}\right) G^{*}\left(x-x^{\prime}, y-y^{\prime}\right) \mathrm{d} x^{\prime} \mathrm{d} y^{\prime}
$$

Numerical evaluation of this integral in each element of the mesh produces the temperature distribution in the resistor. With this information, new values for the electrical conductivity can be determined if the relation $\sigma(T)$ is known. For many materials, the temperature dependence can be linearized with good accuracy in a temperature range around a reference temperature $T_{\text {ref }}$ (usually $300 \mathrm{~K})$. The following form applies:

$$
\sigma(T)=\frac{\sigma_{r e f}}{1+\alpha \Delta T}
$$

in which $\alpha$ is the so called temperature coefficient (in $\left.\mathrm{K}^{-1}\right), \sigma_{\text {ref }}=\sigma\left(T_{\text {ref }}\right)$ and $\Delta T=T-T_{\text {ref }}$. Values for $\sigma_{r e f}$ and $\alpha$ are tabulated and can be found e.g. in [2]. Note that for most metals $\alpha$ is about 0.006 or smaller, however in semiconductors it can be negative and one or several orders of magnitude larger. The $\sigma(x, y)$ distribution obtained from (12) functions as input variable for the electrical solver (see previous section).

One may account for the temperature dependence of the thermal conductivity $k(T)$ in the following, highly approximative way. From the obtained $T(x, y)$ distribution we determine the average source temperature $T_{a v g}$. Note 
this temperature is also useful for calculating the thermal resistance $R_{t h}=\frac{T_{a v g}}{P_{t o t}}$, with

$$
P_{\text {tot }}=\iint_{\text {resistor }} P(x, y) \mathrm{d} x \mathrm{~d} y
$$

the total power dissipated in the resistor. The average temperature in the relevant substrate volume may now be roughly estimated as:

$$
T_{s u b}=\frac{T_{a v g}}{2}
$$

Remind that $T_{\text {sub }}$ is a temperature relative to $T_{\text {ambient }}=$ $T_{\text {ref }}$. Let us now define a perturbation factor $P F$ :

$$
P F=\frac{k\left(T_{r e f}\right)}{k\left(T_{r e f}+T_{s u b}\right)}=\frac{k_{r e f}}{k^{\prime}}
$$

where $k^{\prime}$ can be seen as the average, 'effective' thermal conductivity of the substrate. Since the Green's function is inversely proportional to $k$, the modified temperature field is immediately given by

$$
T^{*}(x, y)=P F \times T(x, y)
$$

$T^{*}$ instead of $T$ is then used to calculate the $\sigma$ distribution.

\section{PRACTICAL EXAMPLES}

\section{Simple Rectangular Resistor}

\section{Setup and parameters}

A resistor of $1 \mathrm{k} \Omega$ to be used in an opamp circuit will be subject to a voltage drop of $15 \mathrm{~V}$. In other words, it is assumed to dissipate $225 \mathrm{~mW}$. The circuit is integrated on a silicon substrate with thickness $t_{s}=300 \mu \mathrm{m}$.

The resistor will be implemented as a n-type silicon layer with thickness $t_{r}=1 \mu \mathrm{m}$ and a doping concentration of $N_{D}=10^{17} \mathrm{~cm}^{-3}$. The electrical conductivity of the layer satisfies:

$$
\sigma(T) \approx q N_{D} \mu_{n}(T)
$$

with $q=1.6 \times 10^{-19} \mathrm{C}$ the elementary charge and $\mu_{n}$ the electron mobility (in $\mathrm{cm}^{2} / \mathrm{Vs}$ ). The temperature dependence of the latter is given by the following empirical fit [3]:

$$
\mu_{n}(T)=\mu_{\min }(T)+\frac{\mu_{0}(T)}{1+\left(\frac{N_{D}}{N_{r e f}(T)}\right)^{a(T)}}
$$

in which

$$
\begin{gathered}
\mu_{\text {min }}=92\left(\frac{T}{300}\right)^{-0.57}, N_{\text {ref }}(T)=1.3 \times 10^{17}\left(\frac{T}{300}\right)^{2.4} \\
\mu_{0}(T)=1268\left(\frac{T}{300}\right)^{-2.33}, a(T)=0.91\left(\frac{T}{300}\right)^{-0.146}
\end{gathered}
$$

Linearization of (17) around an ambient temperature $T_{\text {ref }}=300 \mathrm{~K}$ results in $\sigma_{\text {ref }}=1.3 \times 10^{3} \Omega^{-1} \mathrm{~m}^{-1}$ and $\alpha=0.0043$, which will be used for the electrothermal simulation. With a film thickness of $1 \mu \mathrm{m}, \sigma_{\text {ref }}$ results in $R_{\square}=769 \Omega /$ square, hence an aspect ratio of 1.3 is needed to obtain the requested value of $1 \mathrm{k} \Omega$. A rectangular $26 \mu \mathrm{m} \times 20 \mu \mathrm{m}$ design is proposed, as illustrated in Fig. 2.

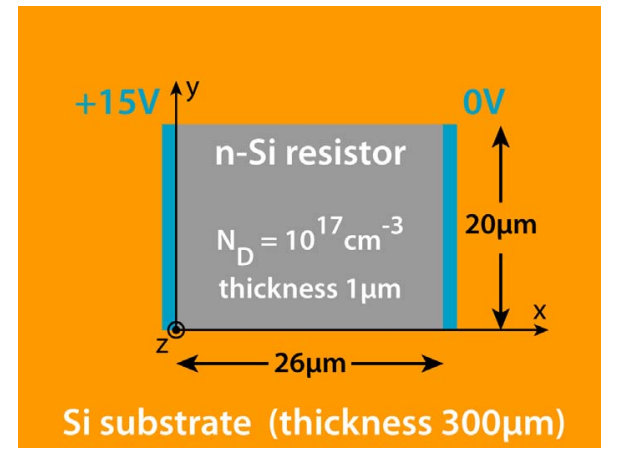

Figure 2. Design for simple $1 \mathrm{k} \Omega$ resistor

The thermal conductivity of the silicon substrate is approximately given by [4, fitted on Fig. 28]:

$$
k_{S i}(T)=\frac{1.13 \times 10^{5}}{T^{1.15}}
$$

This yields $k_{\text {ref }}=160 \mathrm{~W} / \mathrm{mK}$ (at $T_{r e f}=300 \mathrm{~K}$ ) while (15) produces:

$$
P F=\frac{\left(T_{\text {sub }}+300\right)^{1.15}}{300^{1.15}}
$$

As explained before, the thermal solver uses this perturbation factor to approximately correct the temperatures with respect to the modified thermal conductivity.

\section{Results}

The resistor was divided into 68608 triangles for the electrothermal analysis (Fig. 3).

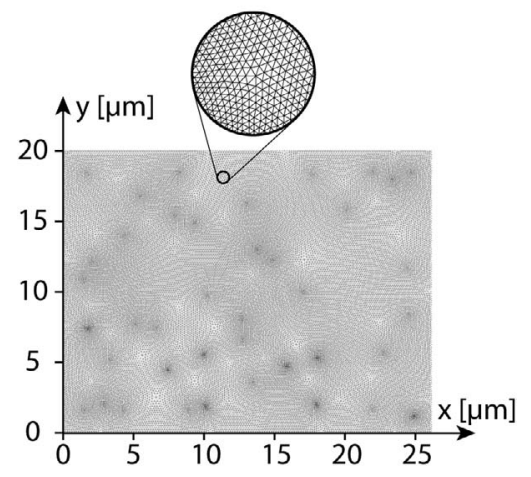

Figure 3. Generated mesh for the resistor design of Fig. 2

The relevant characteristics for the first five iteration stages are summarized in Tables 1 (electrical resistance, power dissipation) and 2 (thermal resistance, temperature range, effective thermal conductivity). The characteristics hardly change during the fifth iteration, hence convergence is reached. Due to the self-heating the element acts as a resistor of $1.115 \mathrm{k} \Omega$, an increase of $11.5 \%$ compared to the original design. The resistor now dissipates $202 \mathrm{~mW}$ and operates at 26.8 degrees on average 
TABLE 1. Electrical characteristics of rectangular n-Si resistor as function of iteration number

\begin{tabular}{ccc}
\hline$\#$ & $\begin{array}{c}R_{e l} \\
{[\Omega]}\end{array}$ & $\begin{array}{c}P_{\text {tot }} \\
{[\mathrm{mW}]}\end{array}$ \\
\hline 1 & 1000.00 & 225.00 \\
2 & 1128.90 & 199.31 \\
3 & 1113.54 & 202.06 \\
4 & 1115.18 & 201.77 \\
5 & 1115.00 & 201.80 \\
\hline
\end{tabular}

TABLE 2. Thermal characteristics of rectangular n-Si resistor as function of iteration number

\begin{tabular}{cccccc}
\hline$\#$ & $\begin{array}{c}R_{t h} \\
{[\mathrm{~K} / \mathrm{W}]}\end{array}$ & $\begin{array}{c}T_{\text {avg }} \\
{[\mathrm{K}]}\end{array}$ & $\begin{array}{c}T_{\min } \\
{[\mathrm{K}]}\end{array}$ & $\begin{array}{c}T_{\max } \\
{[\mathrm{K}]}\end{array}$ & $\begin{array}{c}k_{\text {eff }} \\
{[\mathrm{W} / \mathrm{mK}]}\end{array}$ \\
\hline 1 & 133.31 & 30.00 & 18.30 & 35.65 & 151.70 \\
2 & 132.54 & 26.42 & 16.10 & 31.42 & 152.61 \\
3 & 132.63 & 26.80 & 16.33 & 31.87 & 152.51 \\
4 & 132.62 & 26.76 & 16.31 & 31.82 & 152.52 \\
5 & 132.62 & 26.76 & 16.31 & 31.83 & 152.52 \\
\hline
\end{tabular}

above ambient. An identical simulation but assuming $k$ as a constant $(P F=1)$ produced $R_{e l}=1.110 \mathrm{k} \Omega(-$ $0.4 \%)$ and $T_{a v g}=25.6 \mathrm{~K}$. The temperature dependence of the thermal conductivity therefore seems not very critical with respect to the electrical resistance value.

A designer could try to anticipate the heating process. The average temperature generated by a uniform power dissipation (i.e. $T_{a v g}$ obtained during the first iteration, where electrothermal coupling is not yet established) gives an estimation for the final resistance value:

$$
R_{e l} \approx(1+0.0043 \times 30) \cdot 1 \mathrm{k} \Omega=1.129 \mathrm{k} \Omega
$$

This deviates $14 \Omega(1.3 \%)$ from the correct value, an error which must completely attributed to the non-uniform temperature and power dissipation.

The final distributions for the current density, power dissipation and temperature are shown in Fig. 4. These images can be understood fairly easily. The highest temperatures are found in the middle while zones closer to the edges are cooler (Fig. 4c). This leads to poorer electrical conductivity in the center of the resistor, explaining the higher current density along the sides (Fig. 4a). At the sides, the combination of a smaller local resistance and larger current density gives rise to peak values of the power dissipation (Fig. 4b).

\section{Serpentine Resistor}

\section{Setup and parameters}

A resistor of $4 \Omega$ for an audio amplifier is used to deliver a $2.5 \mathrm{~W}$ signal to the output (voltage drop of $3.2 \mathrm{~V}$ ). We will use an aluminium strip $\left(\sigma_{r e f}=3.77 \times 10^{7} \Omega^{-1} \mathrm{~m}^{-1}\right.$, $\alpha=0.00429)$ for the implementation, with a thickness of $1 \mu \mathrm{m}\left(R_{\square}=26.5 \mathrm{~m} \Omega /\right.$ square $)$. As in the previous example the resistor is integrated on a $300 \mu \mathrm{m}$ silicon substrate, for which (21) will be used again. As the resistance per square is very small, a narrow and long line will be needed (high aspect ratio). A 'serpentine' design (a) Current density $|J(x, y)|$

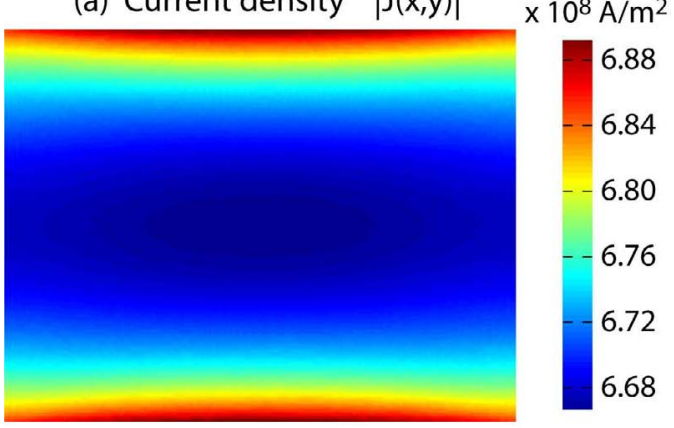

(b) Power density $P(x, y)$

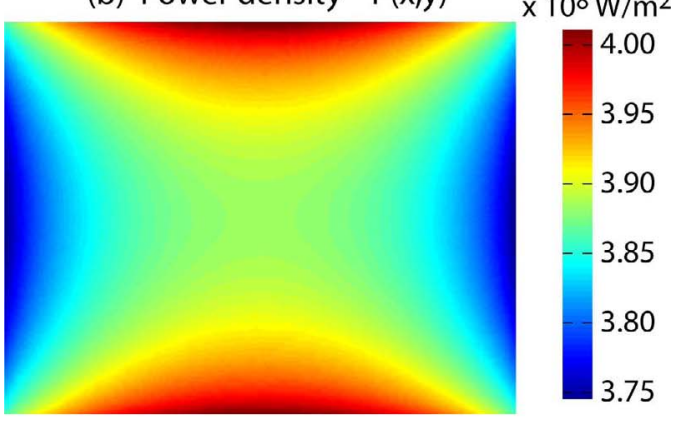

(c) Temperature $T(x, y)$

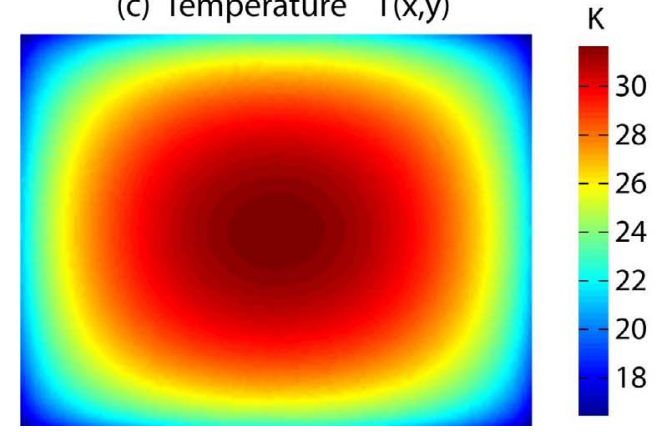

Figure 4. Distributions in rectangular $n$-Si resistor obtained after 5 iterations (convergence reached)

as shown in Fig. 5 is proposed, keeping the resistor compact and saving silicon area.

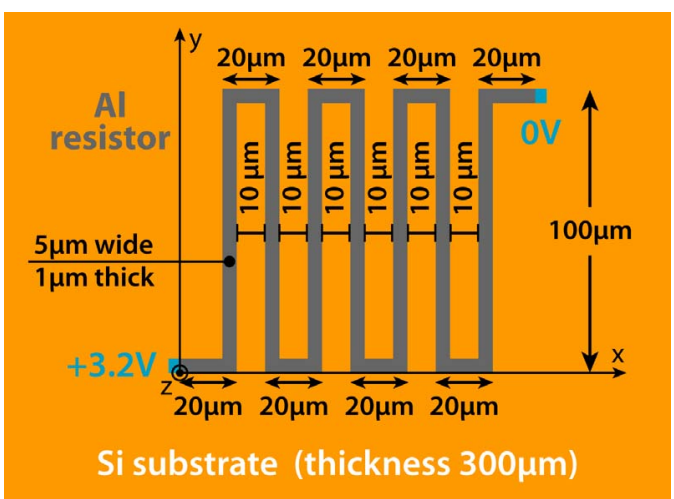

Figure 5. Design for $4 \Omega$ resistor

\section{Results}

A mesh with a total of 25984 triangles was generated for the electrothermal analysis of the resistor. Iteration results for the main electrical and thermal characteristics can be found in Table 3 and 4 respectively. 
TABLE 3. Electrical characteristics of serpentine Al resistor as function of iteration number

\begin{tabular}{ccc}
\hline$\#$ & $R_{e l}$ & $P_{t o t}$ \\
& {$[\Omega]$} & {$[\mathrm{W}]$} \\
\hline 1 & 4.021 & 2.538 \\
2 & 5.384 & 1.895 \\
3 & 5.012 & 2.036 \\
4 & 5.093 & 2.004 \\
5 & 5.074 & 2.011 \\
\hline
\end{tabular}

TABLE 4. Thermal characteristics of serpentine $\mathrm{Al}$ resistor as function of iteration number

\begin{tabular}{cccccc}
\hline$\#$ & $\begin{array}{c}R_{t h} \\
{[\mathrm{~K} / \mathrm{W}]}\end{array}$ & $\begin{array}{c}T_{\text {avg }} \\
{[\mathrm{K}]}\end{array}$ & $\begin{array}{c}T_{\min } \\
{[\mathrm{K}]}\end{array}$ & $\begin{array}{c}T_{\max } \\
{[\mathrm{K}]}\end{array}$ & $\begin{array}{c}k_{\text {eff }} \\
{[\mathrm{W} / \mathrm{mK}]}\end{array}$ \\
\hline 1 & 30.79 & 78.13 & 45.45 & 94.47 & 141.19 \\
2 & 29.96 & 56.79 & 32.10 & 69.77 & 145.51 \\
3 & 30.15 & 61.39 & 34.87 & 75.25 & 144.53 \\
4 & 30.11 & 60.34 & 34.23 & 74.01 & 144.75 \\
5 & 30.12 & 60.58 & 34.38 & 74.30 & 144.70 \\
\hline
\end{tabular}

Again the values are nearly stable after five iterations. The electrical resistance is now $5.074 \Omega$, a $26 \%$ increase compared to the original design. The resistor dissipates $2.011 \mathrm{~W}$ at 60.6 degrees on average above ambient. A simulation with constant thermal conductivity resulted in $R_{e l}=4.991 \Omega$ and $T_{a v g}=55.69 \mathrm{~K}$. So also here the thermal perturbation method is not necessary to obtain a good estimation for the electrical resistance: the deviation from $5.074 \Omega$ is only $1.6 \%$. One could, as explained earlier, try to anticipate the self-heating. The estimation with $T_{a v g}=78.1 \mathrm{~K}$ results in $R_{e l} \approx 5.368 \Omega$, being $5.8 \%$ larger than the correct value.

Let us now focus on the distributions for the current and power densities and the temperature. Fig. 6 shows the calculation results. From Fig. $6 a$ it is clear that in the bends the majority of the current 'cuts the corner'. The outer parts are hardly used and become 'dead' zones. This is a well known phenomenon: a rule of thumb suggests counting the bends only as half a square when designing a serpentine-like resistor. Such a current flow will generate peak values for the power dissipation in a small region near the inner corners, as seen in Fig. 6b. The power distribution is reproduced in Fig. 6c with a different color scale, in order to clarify the moderate values throughout the rest of the resistor. Finally one sees the largest heating takes place in the central part of the resistor (Fig. 6d). This should not be a surprise. The power dissipation is nearly uniform, and the middle part has the largest amount of surrounding heat sources, moreover at relatively small distance. Therefore the temperature is highest in the middle and decreases gradually towards the outer regions.

\section{Thermal Runaway}

\section{Background}

For the last example we will investigate a resistor made of material with a negative temperature coefficent. Note (a) Current density $|J(x, y)|$

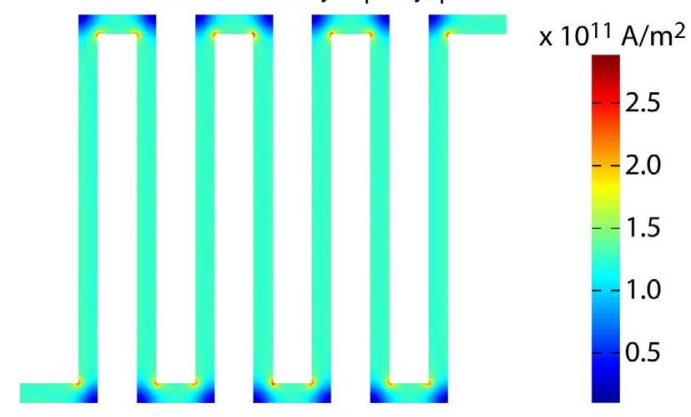

(b) Power density $P(x, y)$

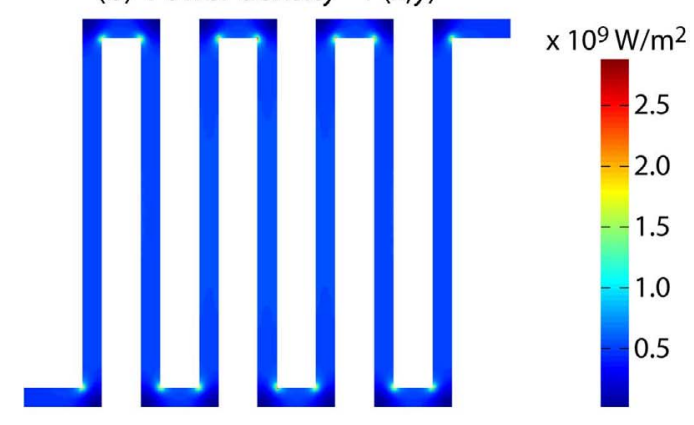

(c) Power density $P(x, y)$

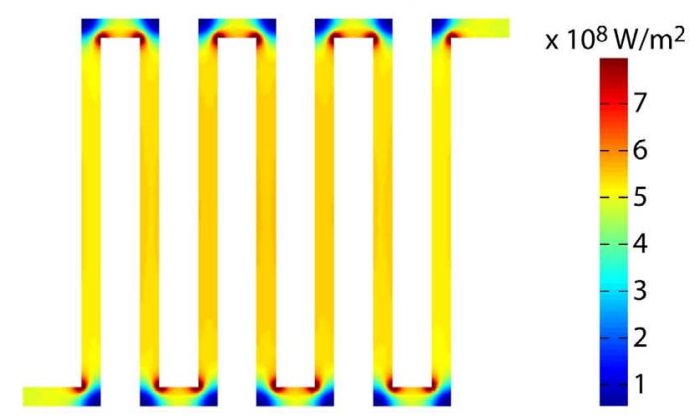

(d) Temperature $T(x, y)$

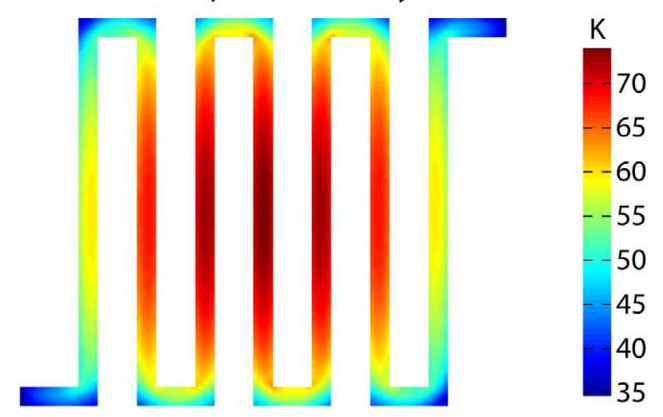

Figure 6. Distributions in serpentine Al resistor obtained after 5 iterations (convergence reached)

that this may occur in intrinsic semiconductor materials, or in devices where the electrical energy is transported by the minority carriers. When $\alpha<0$, the local resistance decreases in hot areas. As a consequence the current will tend to concentrate there, leading to a higher power dissipation and probably a further increase of the temperature. Occurance of such a so called thermal runaway depends on the magnitude of the positive feedback. For moderate magnitudes the current gathering and high power dissipation will be restricted to a relatively small area. This 
tempers the overall temperature increase, explaining the existance of situations which are still stable. When strong feedback occurs the peak temperatures may damage or even entirely destroy the component, commonly known as a thermal breakdown.

\section{Results}

We will illustrate the thermal breakdown mechanism by means of the rectangular resistor design of Fig. 2.

First we analyzed $\alpha=-0.004$. After 8 iterations the following convergent (i.e. the situation is stable) characteristics were obtained: $R_{e l}=859 \Omega, P_{t o t}=262 \mathrm{~mW}$, $T_{a v g}=35.5 \mathrm{~K}, R_{t h}=134.4 \mathrm{~K} / \mathrm{W}$ and $k_{e f f}=150.4$ $\mathrm{W} / \mathrm{mK}$. The distributions were very similar to those shown in Fig. 4 for the 'positive' material.

Various simulations indicated that the resistor stays stable for a temperature coefficient up to -0.0075 . For $\alpha=$ -0.008 the convergence was lost: after 22 iterations a negative electrical resistance was detected. In practice this means the electrical conductivity has passed through infinity - see (12) - which would destroy the component. We would like to stress that from these observations strictly speaking only a numerical instability can be concluded. This does not yet prove that the underlying physical process being modelled is actually unstable. Transient simulations, which are currently in development, could clarify this situation. In Fig. 7 the distributions for power dissipation and temperature are presented for three different iteration stages. The formation of a central highly conducting channel is clearly visible.
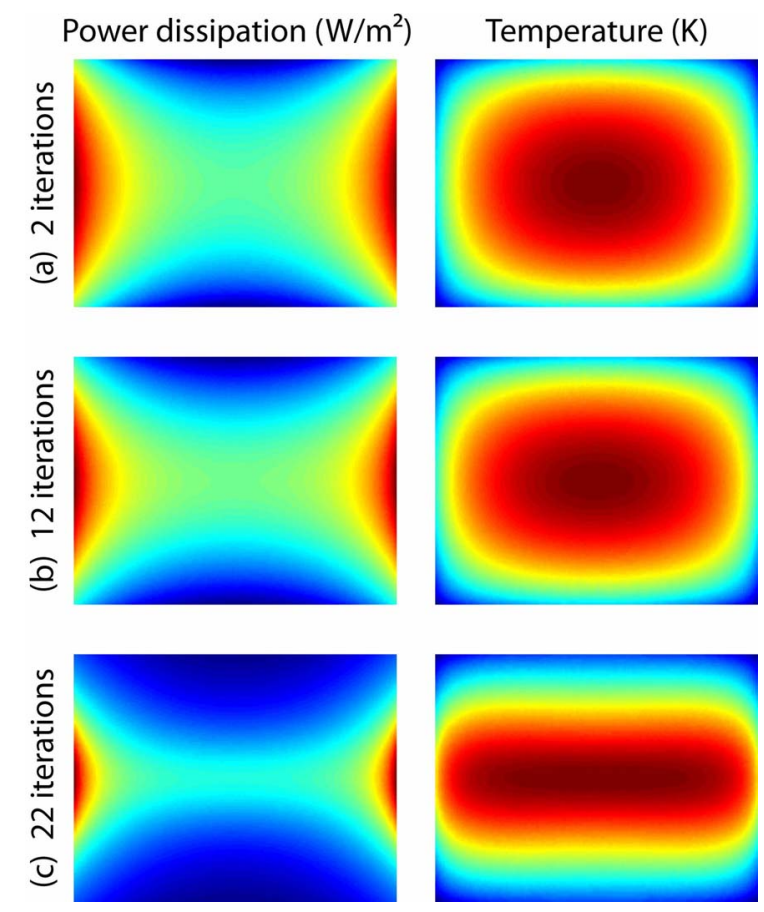

Figure 7. Distributions for rectangular $n$-Si resistor with $\alpha=-0.008$ : (a) initial, (b) feedback in progress, (c) just before breakdown

The upcoming breakdown phenomenon is also detectable by the sudden change of various characteristics near the 22nd iteration, as can be seen in Fig. 8.

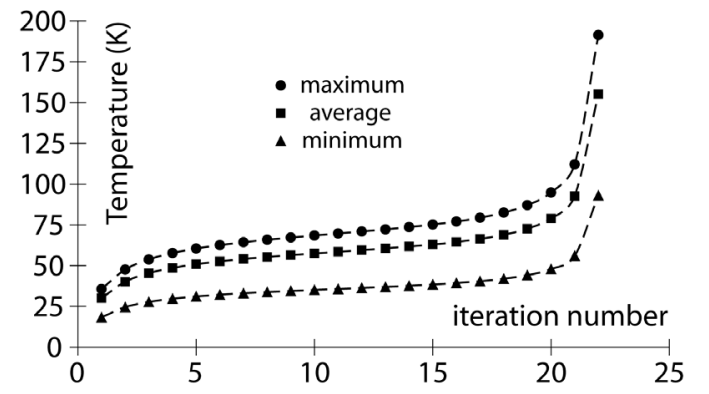

Figure 8. Temperature development for thermal breakdown simulation as function of iteration stage

\section{CONCLUSIONS}

We have presented a method for electrothermal analysis of integrated thin-film resistors. The technique consists of the alternating semi-analytical calculation of the electrical and thermal fields. Hereby we did not turn to the circuit or system level but focused on the device itself. With the proposed method we were able to analyze the electrothermal coupling arising from the self-heating process. Application of the simulator to practical resistor designs produced convergent characteristics after 5 to 10 iterations. The results show the resistor behaviour is clearly different from the original design. Finally we have also investigated a non-stable material to illustrate a thermal runaway and breakdown phenomenon.

\section{THE AUTHORS}

Bjorn Vermeersch and Gilbert De Mey are with the Dept. of Electronics and Information Systems, Ghent University, Sint Pietersnieuwstraat 41, 9000 Gent (Belgium). E-mail addresses: bjorn.vermeersch@elis.ugent.be , demey@elis.ugent.be

\section{ACKNOWLEDGEMENTS}

B. Vermeersch is preparing a $\mathrm{PhD}$ as a Research Assistant for the Research Foundation - Flanders (FWO - Vlaanderen) and wishes to thank FWO for supporting the presented work.

\section{REFERENCES}

[1] M. Kaminski, K. Slusarczyk, A. Napieralski, "Electrothermal simulation of analog and digital integrated circuits", Proc. 12th Int. Conf. on Mixed Design of Integrated Circuits and Systems (MIXDES 2005), pp. 249 - 254, 2005.

[2] D.C. Giancoli, "Physics" (4th Edition), Prentice Hall, 1995.

[3] R.F. Pierret, "Semiconductor Device Fundamentals", Addison-Wesley, p. 83, 1996.

[4] S.M. Sze, "Physics of semiconductor devices" (2nd Edition), Wiley, p. 43, 1981. 